\title{
Antiepileptic Treatment Is Associated with Bone Loss: Difference in Drug Type and Region of Interest
}

\author{
Hooman Salimipour ${ }^{1}$, Sara Kazerooni ${ }^{2}$, Mohammad Seyedabadi $^{3}$, Iraj Nabipour ${ }^{4}$, Reza Nemati ${ }^{1}$, Darioush Iranpour $^{3}$, \\ and Majid Assadi ${ }^{3}$

\begin{abstract}
${ }^{1}$ Department of Neurology, Faculty of Medicine, Bushehr University of Medical Sciences, Bushehr, Iran; ${ }^{2}$ Department of Pediatrics, Faculty of Medicine, Bushehr University of Medical Sciences, Bushehr, Iran; ${ }^{3}$ Persian Gulf Nuclear Medicine Research Center, Bushehr University of Medical Sciences, Bushehr, Iran; and ${ }^{4}$ Persian Gulf Tropical Medicine Research Center, Bushehr University of Medical Sciences, Bushehr, Iran
\end{abstract}

The effects of antiepileptic drugs (AEDs) on bone mineral density (BMD) are well addressed, but data on children, especially concerning new antiepileptic medications, are scarce. This study aimed to reveal the impact of these drugs on the BMD of ambulatory patients with epilepsy. Methods: BMD and detailed clinical information were obtained on 108 patients and 38 controls using dual-energy $x$-ray absorptiometry. The patients were categorized into 2 groups: enzyme-inducing AEDs (EIAEDs) and non-EIAEDs. Also, the patients were classified as being in either a monotherapy or a polytherapy group. All patients completed a 63-item questionnaire. In this study, the raw value of BMD, $T$ score, and $z$ score of the spine, neck of femur, total hip, and forearm were analyzed. Results: Patients receiving AEDs showed diminished lumbar BMD, compared with controls $(P<0.05)$, regardless of the type of AED (EIAEDs or non-EIAEDs). In addition, there was a significant decrease in femoral neck BMD in patients receiving EIAEDs $(0.922 \pm 0.161, P<0.05)$ but not in those treated with non-EIAEDs. Patients undergoing carbamazepine monotherapy (33 patients) showed diminished lumbar and femoral neck BMD, whereas those receiving valproate (22 patients) or undergoing polytherapy showed a BMD similar to that of controls in all studied regions of interest. There was no notable change in BMD in the Ward triangle, trochanter, or total hip in either the EIAED or the non-EIAED group. Conclusion: AED therapy, especially in patients on new-generation medication, is associated with low bone density. It is hoped that the presented data stressing several clinical and diagnostic points will stimulate a high index of suspicion to facilitate early diagnosis and preventive care.

Key Words: bone mineral density (BMD); antiepileptic drugs (AEDs); convulsion; dual-energy $x$-ray absorptiometry (DXA)

J Nucl Med Technol 2013; 41:208-211

DOI: $10.2967 /$ jnmt.113.124685

Received Apr. 10, 2013; revision accepted Jun. 4, 2013.

For correspondence or reprints contact: Majid Assadi, Persian Gulf Nuclear Medicine Research Center, Bushehr University of Medical Sciences, Bushehr 3631, Iran.

E-mail: assadipoya@yahoo.com, asadi@bpums.ac.ir

Published online Jul. 25, 2013.

COPYRIGHT (C 2013 by the Society of Nuclear Medicine and Molecular Imaging, Inc.
D of chronic debilitating diseases such as epilepsy (1). Antiepileptic drugs (AEDs) are associated with decreased bone mineral density (BMD) and increased fracture risk (2). Increased metabolism of 25-hydroxyvitamin D to inactive metabolites after cytochrome p450 induction is thought to be the main mechanism of bone loss by enzyme-inducing AEDs (EIAEDs: phenobarbital, carbamazepine, oxcarbamazepin, phenytoin) (3-6). On the other hand, pregnane $\mathrm{X}$ receptor, a nuclear receptor activated by xenobiotic compounds, including phenytoin phenobarbital and even valproic acid, promotes the expression of vitamin D-responsive genes, including those responsible for vitamin D metabolism $(2,7)$. Bone resorption due to secondary hyperparathyroidism is also demonstrated for phenytoin but not for carbamazepine, valproate, or lamotrigine $(2,8)$. In addition, some studies have not found a significant association between vitamin D, parathyroid hormone, and AEDs $(6,9)$. Inhibition of intestinal absorption of calcium, activation of vitamin D, and an increase in osteoblasts are also reported to play a role in AED-induced bone loss, even non-EIAEDs $(2,10)$. Furthermore, the fact that bone loss does not appear simultaneously in all regions may interfere with the interpretation of BMD data.

In this study, we aimed to investigate the possible effect of antiepileptic treatment on BMD in different regions of interest (ROIs).

\section{MATERIALS AND METHODS}

\section{Participants and Study Design}

Patients receiving different AEDs were studied for BMD. Also, some patients who were taking new-generation antiepileptic medication such as lamotrigine, topiramate, clonazepam, and ethosuximide were included in this study. Overall, the study comprised 108 patients and 38 control subjects. The patients were categorized according to their treatment protocol (monotherapy or polytherapy) and type of drug (enzyme-inducer [phenytoin, phenobarbital, carbamazepine] or non-enzyme inducer [valproate, ethosuximide, lamotrigine, clonazepam]). Patients with hyperparathyroidism, renal failure, or menopause and those undergoing hormone replacement therapy or corticosteroid/anticoagulant therapy were excluded. 
All participants completed a detailed 63-item questionnaire that gathered demographic and behavioral data and asked about a medical history of conditions that could affect bone mass and metabolism. The body mass index is described as the individual's body mass divided by the square of height $\left(\mathrm{kg} / \mathrm{m}^{2}\right)$.

The study complied with the Declaration of Helsinki and was confirmed by the institutional ethics committee of Bushehr University of Medical Sciences.

\section{BMD}

BMD was determined for the spine (L2-L4), greater trochanter, Ward triangle, and femoral neck using dual-energy x-ray absorptiometry (Osteocore II [Medilink] discrepancies). The Ward triangle characterizes the region in the femoral neck with the fewest trabeculae and thus is the area of least BMD. The location of the Ward triangle is patient-specific, and its area is small in comparison to the size of the femur.

World Health Organization criteria were used to determine the ROI $\mathrm{T}$ score (the bone mineral density at the site, compared with the young reference mean). Patients were considered to have normal bone density ( $\mathrm{T}$ score $>-1 \mathrm{SD})$, osteopenia $(-1 \mathrm{SD}<\mathrm{T}$ score $<-2.5 \mathrm{SDs}$ ), or osteoporosis (T score $<-2.5 \mathrm{SDs})$. The nuclear medicine specialist interpreting the results was not aware of the type of protocol or antiepileptic therapy.

\section{Statistical Analysis}

One-way ANOVA was used to compare BMD values in patients receiving monotherapy or polytherapy and enzyme-inducer or nonenzyme-inducer agents. Linear regression analysis was performed to detect the possible influence of different variables on BMD. SPSS software, version 18 (SPSS, Inc.), was used for statistical analysis; the data are presented as mean \pm SEM, and a $P$ value of less than 0.05 was considered significant.

\section{RESULTS}

The data regarding the effects of drug type and protocol on BMD are presented in Tables 1 and 2 .

The study included 108 patients (51 men and 62 women; age, $21.8 \pm 10.31 \mathrm{y}$ ) receiving different AEDs for $43.18 \pm$
30.78 mo. We also included 38 healthy controls ( 8 men and 30 women; age, $30 \pm 6.13 \mathrm{y}$ ).

Patients receiving AEDs showed a diminished lumbar BMD, compared with controls $(P<0.05)$, but there was no significant difference between the groups regarding the type of AED (inducer or noninducer). In addition, there was a significant decrease in femoral neck BMD in patients receiving EIAEDs $(0.922 \pm 0.161, P<0.05)$ but not in those treated with non-EIAEDs.

Individual comparison of the drugs showed that patients receiving carbamazepine (33 patients) had a diminished lumbar BMD $(0.848 \pm 0.213$ vs. $1.029 \pm 0.131, P<0.01)$ and femoral neck BMD $(0.877 \pm 0.174$ vs. $1.004 \pm 0.102$, $P<0.01)$, compared with controls, whereas those receiving valproate (22 patients) showed a BMD similar to that of controls in all studied ROIs. In addition, there was no significant difference between patients undergoing polytherapy and the control group. We could not find a significant difference between the BMD values of patients and controls in the greater trochanter, Ward triangle, or total hip, regardless of type of treatment (EIAEDs or non-EIAEDs) or protocol (monotherapy or polytherapy).

Linear regression analysis revealed that body mass index was positively associated with increased BMD in the lumbar spine $(\beta=0.42, P<0.001)$, femoral neck $(\beta=0.44$, $P<0.001)$, greater trochanter $(\beta=0.46, P<0.001)$, Ward triangle $(\beta=0.21, P<0.05)$, and total hip $(\beta=0.37, P<$ $0.001)$.

We did not find a notable difference in BMD regarding sun exposure. On the other hand, smoking (7 patients) was remarkably associated with a low BMD in the spine $(0.73 \pm$ 0.13 vs. $0.91 \pm 0.20, P<0.05)$, femoral neck $(0.79 \pm 0.15$ vs. $0.93 \pm 0.16, P<0.05)$, greater trochanter $(0.57 \pm 0.08$ vs. $0.91 \pm 0.20, P<0.05)$, and total hip $(0.66 \pm 0.15$ vs. $0.807 \pm 0.19, P<0.05)$

TABLE 1

The Effects of Drug Type on BMD

\begin{tabular}{|c|c|c|c|c|c|}
\hline Region & Group & $n$ & Mean & SD & $95 \% \mathrm{Cl}$ \\
\hline \multirow[t]{3}{*}{ Lumbar spine } & Control & 38 & 1.029 & 0.131 & $0.986-1.072$ \\
\hline & EIAEDs & 72 & $0.906^{*}$ & 0.203 & $0.858-0.954$ \\
\hline & Non-EIAEDs & 36 & $0.905^{\star}$ & 0.215 & $0.832-0.977$ \\
\hline \multirow[t]{3}{*}{ Ward triangle } & Control & 38 & 0.873 & 0.254 & $0.79-0.957$ \\
\hline & EIAEDs & 72 & 0.776 & 0.344 & $0.695-0.856$ \\
\hline & Non-EIAEDs & 36 & 0.772 & 0.375 & $0.645-0.899$ \\
\hline \multirow[t]{3}{*}{ Greater trochanter } & Control & 38 & 0.729 & 0.119 & $0.690-0.769$ \\
\hline & EIAEDs & 72 & 0.682 & 0.134 & $0.651-0.714$ \\
\hline & Non-EIAEDs & 36 & 0.722 & 0.143 & $0.674-0.771$ \\
\hline \multirow[t]{3}{*}{ Femoral neck } & Control & 38 & 1.004 & 0.102 & $0.971-1.038$ \\
\hline & EIAEDs & 72 & $0.922^{*}$ & 0.161 & $0.884-0.96$ \\
\hline & Non-EIAEDs & 36 & 0.946 & 0.164 & $0.890-1.001$ \\
\hline \multirow{3}{*}{ Total hip } & Control & 38 & 0.869 & 0.141 & $0.822-0.915$ \\
\hline & EIAEDs & 72 & 0.793 & 0.184 & $0.75-0.837$ \\
\hline & Non-EIAEDs & 36 & 0.813 & 0.203 & $0.745-0.882$ \\
\hline
\end{tabular}

${ }^{\star} P<0.05$, compared with control.

$\mathrm{Cl}=$ confidence interval. 
TABLE 2

The Effects of Monotherapy or Polytherapy on BMD

\begin{tabular}{llllll}
\hline \multicolumn{1}{c}{ Region } & \multicolumn{1}{c}{ Therapy } & $n$ & Mean & SD & 95\% CI \\
\hline Lumbar spine & Polytherapy & 52 & 0.933 & 0.188 & $0.881-0.985$ \\
& Carbamazepine monotherapy & 33 & $0.848^{*}$ & 0.213 & $0.772-0.924$ \\
& Valproate monotherapy & 22 & 0.917 & 0.231 & $0.814-1.019$ \\
& Control & 38 & 1.0289 & 0.131 & $0.986-1.072$ \\
Ward triangle & Polytherapy & 52 & 0.792 & 0.307 & $0.706-0.877$ \\
& Carbamazepine monotherapy & 33 & 0.744 & 0.394 & $0.604-0.884$ \\
& Valproate monotherapy & 22 & 0.781 & 0.407 & $0.601-0.962$ \\
Greater trochanter & Control & 38 & 0.873 & 0.254 & $0.79-0.957$ \\
& Polytherapy & 52 & 0.708 & 0.140 & $0.669-0.747$ \\
& Carbamazepine monotherapy & 33 & 0.656 & 0.134 & $0.608-0.703$ \\
Femoral neck & Valproate monotherapy & 22 & 0.731 & 0.133 & $0.671-0.79$ \\
& Control & 38 & 0.729 & 0.119 & $0.69-0.769$ \\
& Polytherapy & 52 & 0.938 & 0.1507 & $0.896-0.98$ \\
Total hip & Carbamazepine monotherapy & 33 & $0.887^{\star *}$ & 0.174 & $0.825-0.949$ \\
& Valproate monotherapy & 22 & 0.974 & 0.163 & $0.901-1.046$ \\
& Control & 38 & 1.004 & 0.102 & $0.971-1.038$ \\
& Polytherapy & 52 & 0.813 & 0.161 & $0.768-0.857$ \\
& Carbamazepine monotherapy & 33 & 0.762 & 0.213 & $0.686-0.838$ \\
& Valproate monotherapy & 22 & 0.828 & 0.217 & $0.732-0.925$ \\
& Control & 38 & 0.869 & 0.141 & $0.823-0.915$
\end{tabular}

${ }^{\star} P<0.01$ compared with control.

$\mathrm{Cl}=$ confidence interval.

\section{DISCUSSION}

We observed a significant decrease in lumbar BMD among patients receiving AEDs regardless of the inducer or noninducer drug. Although EIAEDs enhance vitamin D metabolism to inactive metabolites (3-6), non-EIAEDs may also contribute to bone loss by interfering with pregnane $\mathrm{X}$ receptor, intestinal absorption of calcium, activation of vitamin $\mathrm{D}$, osteoblast cell growth, or homocysteine accumulation $(2,10,11)$. Boluk et al. reported that long-term $(>1 \mathrm{y})$ monotherapy of epileptic adults with valproate reduces BMD. They have also demonstrated an increase in the serum levels of parathyroid hormone, phosphorus, and alkaline phosphatase (12).

In addition, a decline in femoral neck BMD was seen in patients treated with EIAEDs but not in those receiving non-EIAEDs. Stephen et al. reported a reduction in femoral neck BMD in 78 epileptic patients receiving EIAEDs (carbamazepine and phenytoin) or non-EIAEDs (mainly valproate), including 47 postmenopausal women (13). Moreover, Pack et al. described a $2.6 \%$ decline of BMD in the femoral neck but not in the lumbar spine or total hip of 93 premenopausal women receiving AEDs for $1 \mathrm{y}(8)$.

There was no notable change in BMD in the Ward triangle, trochanter, or total hip in either the EIAED group or the non-EIAED group. These observations suggest that one ROI may show bone resorption whereas the other ROIs represent normal BMD. Moreover, compiling the data on the femoral neck, greater trochanter, and Ward triangle into total-hip BMD may mask the alterations of BMD in these regions. In this regard, Selby et al. reported that the error associated with total-hip BMD derived from the hip subregions (59\%) is too high to allow clinically meaningful conclusions (14). Total-hip calibration formulas can also influence BMD differences between the different scanners in hip subregions. Therefore, subregion calibration formulas should be applied for calibration of absolute changes (15).

Patients undergoing carbamazepine monotherapy (33 patients) showed diminished lumbar and femoral neck BMD, whereas those receiving valproate (22 patients) or undergoing polytherapy showed BMD similar to that of controls in all studied ROIs. Chou et al. have also demonstrated diminished bone density in children receiving carbamazepine monotherapy, compared with those undergoing valproate monotherapy (16).

We also studied patients receiving lamotrigine, topiramate, clonazepam, and ethosuximide. However, they were also treated with one of the conventional drugs. Therefore, we could not distinguish the main effect of these agents on bone density. In this regard, a retrospective cohort study has found that newer non-EIAEDs (gabapentin, lamotrigine, and topiramate) are less likely to be associated with osteoporosis at the lumbar spine, femoral neck, and total hip (17).

Treatment with more than one AED and with EIAEDs is considered to be more commonly associated with a risk of fracture in postmenopausal women (18). In contrast, combination therapy did not result in reduced BMD in any of the studied ROIs in our study. This inconsistency may result from the difference in population or the type of drug combination.

In contrast to Andress et al., who reported age and duration of treatment as critical risk factors for low BMD in men receiving AEDs (19), we observed that neither age nor duration of AED treatment significantly influenced BMD. 
This controversy may result from the fact that we included both male and female patients. Moreover, most of our patients underwent combination therapy.

Smoking was notably associated with low bone density in the spine, femoral neck, greater trochanter, and total hip. This finding suggests that alteration of lifestyle may help improve bone health in patients receiving AEDs. However, Lyngstad-Brechan reported a diminished mean BMD at the proximal forearm and femoral neck of postmenopausal women receiving anticonvulsants, compared with an age- and lifestyle-matched (smoking habits, physical activity) control group (20). Likewise, an acceleration of bone loss with anticonvulsants has been found even after adjustment for age, smoking, body mass index, health status, and calcium and vitamin D intake (hip BMD loss, $1.16 \%$ in $1 \mathrm{y}$ vs. $0.70 \%$ in controls) (21). These observations suggest that prevention of bone loss in epileptic patients requires more serious clinical management (22).

\section{CONCLUSION}

Antiepileptic treatment, especially carbamazepine monotherapy, is associated with decreased BMD. This decrease of BMD may not appear simultaneously in all regions on dualenergy x-ray absorptiometry scans, suggesting that scanning for BMD be repeated in several ROIs in clinical evaluations.

\section{DISCLOSURE}

This study is the thesis of Dr. Sara Kazerooni and was carried out with the sponsorship of Bushehr University of Medical Sciences (grant 1249). No other potential conflict of interest relevant to this article was reported.

\section{ACKNOWLEDGMENTS}

We thank our colleagues at our institutes for technical help and data acquisition.

\section{REFERENCES}

1. Mazziotti G, Canalis E, Giustina A. Drug-induced osteoporosis: mechanisms and clinical implications. Am J Med. 2010;123:877-884.
2. Lee RH, Lyles KW, Colon-Emeric C. A review of the effect of anticonvulsant medications on bone mineral density and fracture risk. Am J Geriatr Pharmacother. 2010;8:34-46.

3. Dent CE, Richens A, Rowe DJ, Stamp TC. Osteomalacia with long-term anticonvulsant therapy in epilepsy. BMJ. 1970;4:69-72.

4. Hahn TJ, Hendin BA, Scharp CR, Haddad JG Jr. Effect of chronic anticonvulsant therapy on serum 25-hydroxycalciferol levels in adults. N Engl J Med. 1972; 287:900-904.

5. Hahn TJ, Birge SJ, Scharp CR, Avioli LV. Phenobarbital-induced alterations in vitamin D metabolism. J Clin Invest. 1972;51:741-748.

6. Mintzer S, Boppana P, Toguri J, DeSantis A. Vitamin D levels and bone turnover in epilepsy patients taking carbamazepine or oxcarbazepine. Epilepsia. 2006;47: 510-515.

7. Cerveny L, Svecova L, Anzenbacherova E, et al. Valproic acid induces CYP3A4 and MDR1 gene expression by activation of constitutive androstane receptor and pregnane X receptor pathways. Drug Metab Dispos. 2007;35:1032-1041.

8. Pack AM, Morrell MJ, Randall A, McMahon DJ, Shane E. Bone health in young women with epilepsy after one year of antiepileptic drug monotherapy. Neurology. 2008;70:1586-1593.

9. Filardi S, Guerreiro CA, Magna LA, Marques Neto JF. Bone mineral density, vitamin D and anticonvulsant therapy. Arq Neuropsiquiatr. 2000;58:616-620.

10. Sato Y, Kondo I, Ishida S, et al. Decreased bone mass and increased bone turnover with valproate therapy in adults with epilepsy. Neurology. 2001;57:445-449.

11. Elliott JO, Jacobson MP, Haneef Z. Homocysteine and bone loss in epilepsy. Seizure. 2007;16:22-34.

12. Boluk A, Guzelipek M, Savli H, Temel I, Ozisik HI, Kaygusuz A. The effect of valproate on bone mineral density in adult epileptic patients. Pharmacol Res. 2004;50:93-97.

13. Stephen LJ, McLellan AR, Harrison JH, et al. Bone density and antiepileptic drugs: a case-controlled study. Seizure. 1999;8:339-342.

14. Selby PL, McCloskey EV, Robinson J, et al. Is a calculated total hip BMD of clinical use? Osteoporosis International. 2000;11:368-371.

15. Lu Y, Fuerst T, Hui S, Genant HK. Standardization of bone mineral density at femoral neck, trochanter and Ward's triangle. Osteoporosis International. 2001;12: 438-444.

16. Chou IJ, Lin KL, Wang HS, Wang CJ. Evaluation of bone mineral density in children receiving carbamazepine or valproate monotherapy. Acta Paediatr Taiwan. 2007;48:317-322.

17. Lee RH, Lyles KW, Sloane R, Colon-Emeric C. The association of newer anticonvulsant medications and bone mineral density. Endocr Pract. September 14, 2012 [Epub ahead of print].

18. Carbone LD, Johnson KC, Robbins J, et al. Antiepileptic drug use, falls, fractures, and BMD in postmenopausal women: findings from the women's health initiative (WHI). J Bone Miner Res. 2010;25:873-881.

19. Andress DL, Ozuna J, Tirschwell D, et al. Antiepileptic drug-induced bone loss in young male patients who have seizures. Arch Neurol. 2002;59:781-786.

20. Lyngstad-Brechan MA, Tauboll E, Nakken KO, et al. Reduced bone mass and increased bone turnover in postmenopausal women with epilepsy using antiepileptic drug monotherapy. Scand J Clin Lab Invest. 2008;68:759-766.

21. Ensrud KE, Walczak TS, Blackwell T, Ensrud ER, Bowman PJ, Stone KL. Antiepileptic drug use increases rates of bone loss in older women: a prospective study. Neurology. 2004;62:2051-2057.

22. Elliott JO. Possible methods for the prevention of bone loss in persons with epilepsy. Expert Rev Neurother. 2009;9:797-812. 